OPEN ACCESS

Edited by:

Tong-Chuan He,

University of Chicago Medicine,

United States

Reviewed by:

Bao-Liang Song,

Wuhan University, China

Ahm Khurshid Alam,

University of Rajshahi, Bangladesh

*Correspondence:

Takatoshi Anno

anno-t@umin.ac.jp

Specialty section:

This article was submitted to Pharmacology of Anti-Cancer Drugs,

a section of the journal

Frontiers in Oncology

Received: 05 February 2021 Accepted: 14 April 2021

Published: 04 May 2021

Citation:

Anno T, Yamatsuji T, Tomoda K, Nakanishi S and Kaneto H (2021) Case

Report: Various Abnormalities in Lipid and Glucose Metabolism Induced by Capecitabine.

Front. Oncol. 11:664475. doi: 10.3389/fonc.2021.664475

\section{Case Report: Various Abnormalities in Lipid and Glucose Metabolism Induced by Capecitabine}

\author{
Takatoshi Anno ${ }^{1 *}$, Tomoki Yamatsuji ${ }^{2}, K_{\text {Koichi Tomoda }}{ }^{1}$, Shuhei Nakanishi ${ }^{3}$ \\ and Hideaki Kaneto ${ }^{3}$ \\ ${ }^{1}$ Department of General Internal Medicine 1, Kawasaki Medical School, Okayama, Japan, 2 Department of Surgery, \\ Kawasaki Medical School, Okayama, Japan, ${ }^{3}$ Department of Diabetes, Endocrinology and Metabolism, Kawasaki Medical \\ School, Kurashiki, Japan
}

Capecitabine has been used for the treatment of various types of tumors. The rare side effects induced by capecitabine have been reported as hypertriglyceridemia, acute pancreatitis associated with hypertriglyceridemia and hypertriglyceridemia complicated with hyperglycemia. The mechanisms of capecitabine-induced hypertriglyceridemia are unclear. In this report, we present a subject with sigmoid colon cancer and capecitabineinduced dyslipidemia. LDL-cholesterol level was markedly elevated throughout the long period of treatment with capecitabine. In addition, triglyceride level was high and not stable during the treatment period. Her dyslipidemia was ameliorated by the treatment with $5 \mathrm{mg}$ of rosuvastatin, which is one of the HMG-CoA reductase inhibitors.

Keywords: capecitabine, metabolic disorder, dyslipidemia, hypertriglyceridemia, hypercholesterolemia, hyperinsulinemia

\section{BACKGROUND}

Capecitabine $\left(\mathrm{XELODA}^{\circledR}\right)$ has been used for the treatment of various types of tumors, such as breast, colorectal, gastric, head and neck carcinoma. Capecitabine is an oral fluoropyrimidine which is converted to 5-fluorouracil (5-FU) inside tumor cells in vivo, resulting in high drug concentrations. The rare side effects induced by capecitabine have been reported as follows: hypertriglyceridemia (1-9) and acute pancreatitis associated with hypertriglyceridemia (10-12), although the frequent adverse events of capecitabine are diarrhea, hand-foot syndrome, hyperbilirubinemia, and lymphopenia. The mechanisms of capecitabine-induced hypertriglyceridemia are unclear. Moreover, capecitabine-induced hypertriglyceridemia is sometimes complicated with hyperglycemia (13-15). In this report, we present a subject with sigmoid colon cancer and capecitabine-induced dyslipidemia. Low-density lipoprotein (LDL)cholesterol level was markedly elevated throughout the long period of treatment with capecitabine. In addition, triglyceride level was high and not stable during the treatment period. Her dyslipidemia was ameliorated by the treatment with $5 \mathrm{mg}$ of rosuvastatin, which is one of the 3-hydroxy-3methylglutaryl coenzyme A (HMG-CoA) reductase inhibitors. 


\section{CASE PRESENTATION}

A 52-year-old Japanese woman was referred and brought to an emergency room with symptoms of fever and abdominal pain. Her height, body weight and body mass index were $154.2 \mathrm{~cm}$, $66.4 \mathrm{~kg}$ and $27.9 \mathrm{~kg} / \mathrm{m}^{2}$, respectively. She had no remarkable past history and family history. Her vital signs were as follows: temperature, $37.7^{\circ} \mathrm{C}$; blood pressure, $108 / 74 \mathrm{mmHg}$; heart rate, 108 beats/min; oxygen saturation, 95\% (room air). Table 1 shows laboratory data in an emergency room. Her infection markers were markedly elevated: white blood cell, $22850 / \mu \mathrm{L}$ (neutrophil, 93.6\%); C-reactive protein, $17.38 \mathrm{mg} / \mathrm{dL}$; procalcitonin, $69.58 \mathrm{ng} / \mathrm{mL}$. Renal function was almost within the normal range, but liver dysfunction was observed: asparate aminotransferase (AST), $39 \mathrm{U} / \mathrm{L}$; alanine transaminase (ALT), $31 \mathrm{U} / \mathrm{L}$; alkaline phosphatase (ALP), $517 \mathrm{U} / \mathrm{L} ; \gamma$-glutamyl transpeptidase $(\gamma$-GTP), $117 \mathrm{U} / \mathrm{L}$; lactate dehydrogenase $(\mathrm{LDH}), 276 \mathrm{U} / \mathrm{L}$. In addition, her diabetes-associated data were as follows: plasma glucose, $419 \mathrm{mg} / \mathrm{dL}$; hemoglobin $\mathrm{A} 1 \mathrm{c}$ (HbA1c), 9.0\%; plasma insulin $16.4 \mu \mathrm{U} / \mathrm{mL}$. Lipid-associated data were almost within the normal range (total cholesterol, 129 mg/dL; Low Density Lipoprotein (LDL)-cholesterol, 89 mg/dL;
High Density Lipoprotein (HDL)-cholesterol, $15 \mathrm{mg} / \mathrm{dL}$; triglyceride, $102 \mathrm{mg} / \mathrm{dL}$ ). Abdominal computed tomography (CT) and enhanced abdominal CT revealed a tumor in the sigmoid colon lesion, gastrointestinal perforation with free air, ascites and peritonitis. She immediately underwent emergency surgery and was diagnosed as sigmoid colon cancer, invasion to the abdominal wall and uterus and gastrointestinal perforation in the lesion of sigmoid colon cancer. Histopathological diagnosis was adenocarcinoma, and genomic analysis revealed the presence of activating mutations in the KRAS genes. Although during hospitalization period we treated her with intensive insulin therapy to obtain good glycemic control, she took $500 \mathrm{mg} /$ day of metformin at discharge. At that time, her diabetes and dyslipidemia-associated data were as follows: plasma glucose, $145 \mathrm{mg} / \mathrm{dL}$; HbA1c, 7.5\%; total cholesterol, 256 mg/dL; LDL-cholesterol, 140 mg/dL; HDL-cholesterol, 28 $\mathrm{mg} / \mathrm{dL}$; triglyceride, $412 \mathrm{mg} / \mathrm{dL}$. After emergency surgery, we started chemotherapy with $205 \mathrm{mg} \times 1$ day of oxaliplatin and $3,000 \mathrm{mg} \times 14$ days of capecitabine every 3 weeks. Since after 3 courses of this chemotherapy she suffered from bone marrow suppression, we decreased $80 \%$ dose of capecitabine as $2,400 \mathrm{mg} \times 14$ days and continued for more 3 courses of

TABLE 1 | Laboratory data in an emergency room in this subject.

\begin{tabular}{|c|c|c|c|c|c|}
\hline Variable & Result & Reference range & Variable & Result & Reference range \\
\hline \multicolumn{3}{|c|}{ Peripheral blood } & \multicolumn{3}{|c|}{ Diabetes and Dyslipidemia marker } \\
\hline 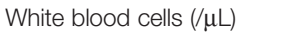 & 22850 & $3300-8600$ & Plasma glucose (mg/dL) & 419 & \\
\hline Neutrophil (\%) & 93.6 & $52.0-80.0$ & Hemoglobin A1c (\%) & 9.0 & $4.9-6.0$ \\
\hline Red blood cells $\left(\times 10^{4} / \mu \mathrm{L}\right)$ & 330 & $386-492$ & Total cholesterol (mg/dL) & 129 & $142-248$ \\
\hline Hemoglobin (g/dL) & 8.6 & $11.6-14.8$ & LDL cholesterol (mg/dL) & 89 & $65-139$ \\
\hline Hematocrit (\%) & 26.1 & $35.1-44.4$ & HDL cholesterol (mg/dL) & 15 & $40-103$ \\
\hline Platelets $\left(\times 10^{4} / \mu \mathrm{L}\right)$ & 62.6 & $15.8-34.8$ & Triglyceride (mg/dL) & 102 & $30-149$ \\
\hline \multicolumn{3}{|c|}{ Blood biochemistry } & \multicolumn{3}{|c|}{ Infectious marker } \\
\hline Total protein (g/dL) & 6.8 & $6.6-8.1$ & $\mathrm{CRP}(\mathrm{mg} / \mathrm{dL})$ & 17.38 & $<0.14$ \\
\hline Albumin (g/dL) & 2.6 & $4.1-5.1$ & Procalcitonin (ng/mL) & 69.58 & $0.00-0.05$ \\
\hline Globulin (g/dL) & 4.2 & $2.2-3.4$ & \multicolumn{3}{|c|}{ Blood Gas Analysis } \\
\hline Total bilirubin (mg/dL) & 0.7 & $0.4-1.5$ & $\mathrm{pH}$ & 7.510 & $7.360-7.460$ \\
\hline AST (U/L) & 39 & $13-30$ & $\mathrm{PCO}_{2}(\mathrm{mmHg})$ & 30.5 & $34.0-46.0$ \\
\hline ALT (U/L) & 31 & $7-23$ & $\mathrm{PO}_{2}(\mathrm{mmHg})$ & 164.0 & $80.0-90.0$ \\
\hline LDH (U/L) & 276 & $124-222$ & $\mathrm{HCO}_{3}^{-}(\mathrm{mEq} / \mathrm{L})$ & 24.1 & $24.0-32.0$ \\
\hline ALP (U/L) & 517 & $106-322$ & $\mathrm{BE}(\mathrm{mEq} / \mathrm{L})$ & 1.7 & $-2.5-2.5$ \\
\hline$\gamma$-GTP (U/L) & 117 & $9-32$ & $\mathrm{SO}_{2}(\%)$ & 99.2 & $95.0-98.0$ \\
\hline BUN (mg/dL) & 6 & $8-20$ & Lactate (mEq/L) & 1.10 & $0.63-2.44$ \\
\hline Creatinine (mg/dL) & 0.56 & $0.46-0.79$ & \multicolumn{3}{|c|}{ Coagulation fibrinolytic system-related antibodies } \\
\hline Cholinesterase (U/L) & 245 & $201-421$ & & & \\
\hline Uric acid (mg/dL) & 2.9 & $2.6-5.5$ & PT-sec (sec) & 15.5 & $9.3-12.5$ \\
\hline Creatine Kinase (U/L) & 27 & $41-153$ & PT-INR & 1.30 & $0.85-1.13$ \\
\hline Amylase $(\mu \mathrm{g} / \mathrm{dL})$ & 25 & $44-132$ & PT-activity (\%) & 58.4 & $80.7-125.2$ \\
\hline Sodium (mmol/L) & 131 & $138-145$ & APTT (sec) & 35.3 & $26.9-38.1$ \\
\hline Potassium (mmol/L) & 3.6 & $3.6-4.8$ & D-dimer $(\mu \mathrm{g} / \mathrm{mL})$ & 2.40 & $<1.0$ \\
\hline Chloride (mmol/L) & 93 & $101-108$ & & & \\
\hline
\end{tabular}


chemotherapy. Chemotherapy was effective for her tumor, and residual sigmoid colon cancer was not detected on positron emission tomography (PET)-CT. Therefore, she was once observed without chemotherapy including capecitabine.

After emergency surgery and through the period of chemotherapy, she was treated with $500 \mathrm{mg} /$ day of metformin and her glycemic control was good (HbAlc: 6.2 $-6.7 \%)$. However, her triglyceride level was elevated before the chemotherapy and was not stable during the period of chemotherapy. In addition, her LDL-cholesterol level was markedly elevated during the period of chemotherapy including capecitabine. We examined fasting data about dyslipidemia in more detail before starting course 6 of chemotherapy. Laboratory data about dyslipidemia were as follows (Table 2): total cholesterol, $312 \mathrm{mg} / \mathrm{dL}$; LDLcholesterol, $196 \mathrm{mg} / \mathrm{dL}$; HDL-cholesterol, $46 \mathrm{mg} / \mathrm{dL}$; triglyceride, $316 \mathrm{mg} / \mathrm{dL}$; remnant-like particle (RLP)cholesterol, $17.0 \mathrm{mg} / \mathrm{dL}$; lipoprotein lipase (LPL), $95 \mathrm{ng} / \mathrm{mL}$; apolipoprotein A-I, $126 \mathrm{mg} / \mathrm{dl}$; apolipoprotein A-II, $26.1 \mathrm{mg} / \mathrm{dl}$; apolipoprotein B, $181 \mathrm{mg} / \mathrm{dl}$; apolipoprotein C-II, $9.8 \mathrm{mg} / \mathrm{dl}$; apolipoprotein C-III, $23.7 \mathrm{mg} / \mathrm{dl}$; apolipoprotein E, $10.7 \mathrm{mg} / \mathrm{dl}$. In addition, lipoprotein fractions showed very low density lipoprotein (VLDL) and midband fractions were high (VLDL, 21\%; midband, 39\%). As shown in Table 2, LDLcholesterol level during the period of chemotherapy (196-214 $\mathrm{mg} / \mathrm{dL}$ ) was markedly higher compared to before (80-140 $\mathrm{mg} / \mathrm{mL}$ ). LPL level was as low as $95 \mathrm{ng} / \mathrm{mL}$ (reference range $164-284 \mathrm{ng} / \mathrm{mL}$ ) after course 5 of the chemotherapy including capecitabine. Fasting laboratory data about diabetes were as follows; plasma glucose, $128 \mathrm{mg} / \mathrm{dL}$; plasma insulin, $27.5 \mu \mathrm{U} / \mathrm{mL}$; HbA1c, 6.3\%; glycoalbumin $15.1 \%$. As shown in Table 2, plasma insulin level after starting the chemotherapy $(27.5 \mu \mathrm{U} / \mathrm{mL})$ was markedly higher compared to before $(16.4 \mu \mathrm{U} / \mathrm{mL})$.

After then, we started $5 \mathrm{mg} /$ day of rosuvastatin for the treatment of dyslipidemia, and after then LDL-cholesterol level was decreased to $110 \mathrm{mg} / \mathrm{dL}$ and continued to be within normal range. VLDL and midband fractions on lipoprotein fractions were markedly decreased (VLDL, from $21 \%$ to $13 \%$; midband, from 39\% to 28\%). Furthermore, as shown in Figure 1, her hyperinsulinemia was markedly improved (plasma insulin, $13.5 \mu \mathrm{U} / \mathrm{mL}$ ) compared to that during the chemotherapy (25.1-27.5 $\mu \mathrm{M} / \mathrm{mL}$ ), although plasma insulin level was still slightly higher compared to its reference range presumably due to overweight.

\section{DISCUSSION}

Herein, we report a case of sigmoid colon cancer and capecitabine-induced dyslipidemia (increase of LDLcholesterol, reduction of LPL activity) and metabolic disorder (hyperinsulinemia). It is known that hypertriglyceridemia is sometimes induced by capecitabine, although the mechanisms of capecitabine-induced hypertriglyceridemia are unclear. In this subject, however, LDL-cholesterol was markedly elevated through the treatment with capecitabine. Her dyslipidemia was

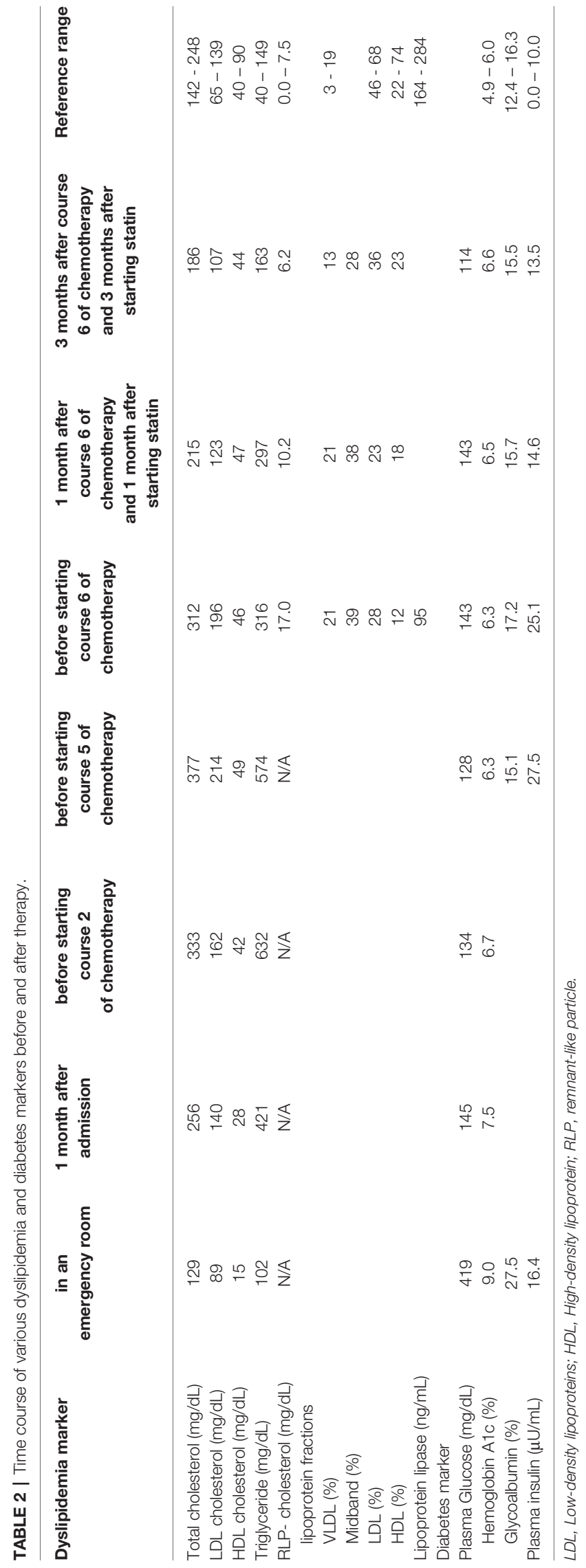




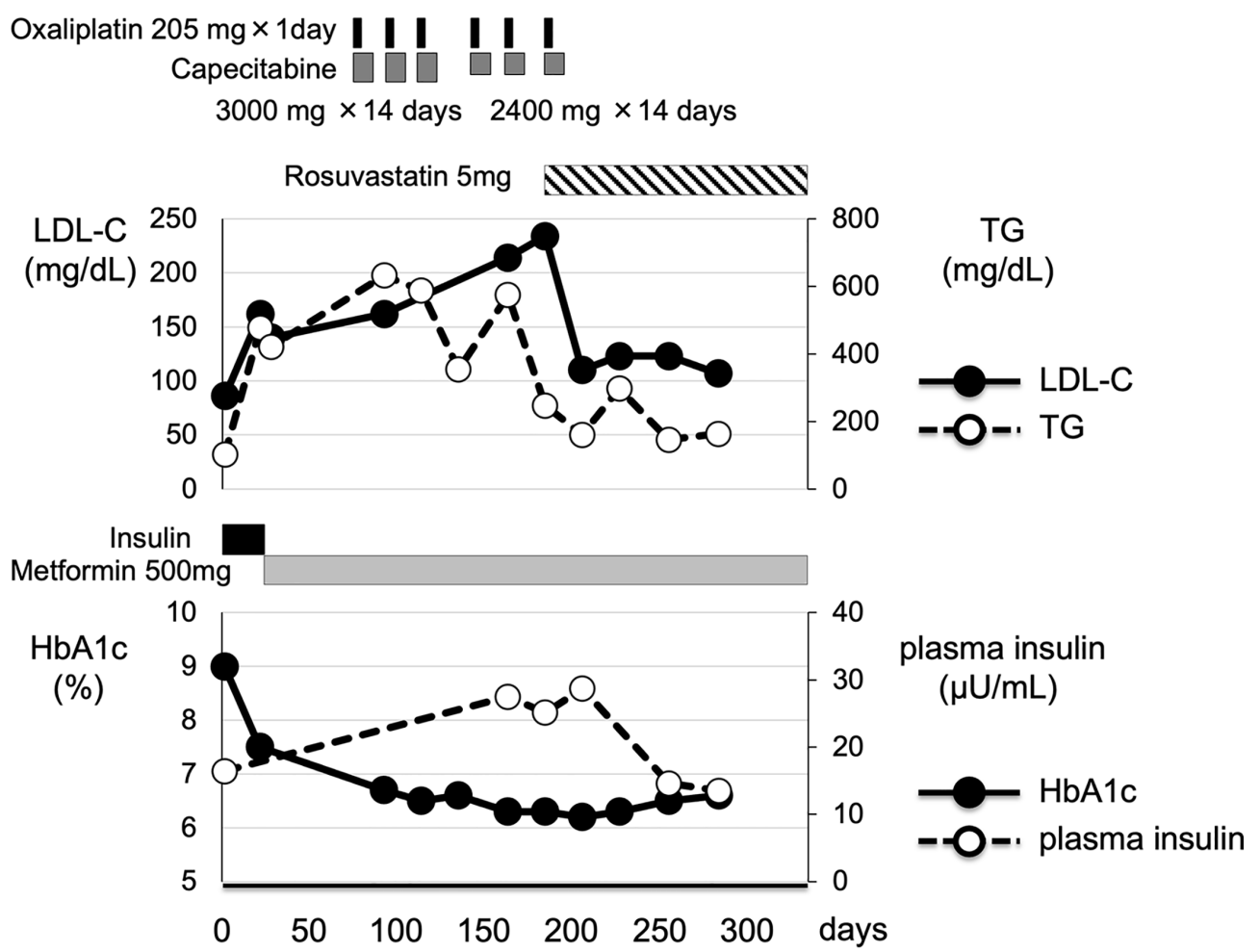

FIGURE 1 | Clinical time course of clinical parameters in this subject. After emergency surgery, this patient underwent 6 courses of chemotherapy including capecitabine. After emergency surgery, she was treated with 500 mg of metformin for diabetes mellitus. In addition, through the chemotherapy including capecitabine, her plasma insulin level and LDL-cholesterol level were markedly elevated. And then, she was treated with 5 mg of rosuvastatin, one of the HMG-CoA reductases, for the treatment of dyslipidemia. Her plasma insulin and LDL-cholesterol level were markedly decreased.

well treated with an HMG-CoA reductase inhibitor throughout the period of the treatment with capecitabine and improved after the end of chemotherapy.

Our patient had hypertriglyceridemia and type 2 diabetes mellitus just after emergency surgery and before the chemotherapy including capecitabine. However, her plasma insulin level and LDL-cholesterol level were markedly elevated during the period of chemotherapy including capecitabine, although her glycemic control was good with the treatment of metformin therapy. Insulin resistance is caused by metabolic syndrome and various metabolic disorders especially in obese subjects. In addition, it is known that the decreased insulin effect, which is induced by insulin resistance, leads to the reduction of LPL activity (16). Her LPL level was as low as $95 \mathrm{ng} / \mathrm{mL}$ (reference range $164-284 \mathrm{ng} / \mathrm{mL}$ ) after course 5 of the chemotherapy including capecitabine, and her insulin level was markedly elevated at that time, although her glycemic control continued to be good. In addition, she had both hypercholesterolemia and hypertriglyceridemia. Her triglyceride level was high before the chemotherapy, and LDL-cholesterol level was markedly elevated during the period of capecitabine therapy. Her dyslipidemia was well treated with an HMG-CoA reductase inhibitor and improved after the end of chemotherapy including capecitabine. Her RLPcholesterol, which is known to stagnate the metabolism of triglyceride, was increased. RLP-cholesterol is elevated in patients with metabolic syndrome (17). And increased remnant lipoprotein leads to an abnormal concentration of triglyceride, and lipoprotein fractions show elevation of VLDL and midband (18). Her plasma insulin level was markedly elevated during the period of chemotherapy including capecitabine These data suggest that she was under metabolic disorder conditions during the period. In addition, her RLP-cholesterol level and VLDL and midband lipoprotein fractions were elevated presumably due to decreased LPL activity during the period of chemotherapy. In such conditions, the patient was exposed to hypercholesterolemia and hypertriglyceridemia probably together with insulin resistance. There were several reports showing capecitabine-induced hypertriglyceridemia, capecitabineinduced hypertriglyceridemia complicated with acute pancreatitis and capecitabine-induced hypertriglyceridemia complicated with hyperglycemia. We think that such reported side effect is one of a wide range of capecitabine-induced abnormalities in lipid and glucose metabolism in the whole body. The mechanism of capecitabine-induced various abnormalities in lipid and glucose metabolism remains unclear, although capecitabine-induced abnormalities are usually accompanied by mixed disturbance of the metabolic profiles. However, various abnormalities of our patient profile clearly indicated that capecitabine-induced metabolic disorder was associated with hyper insulinemia and insulin resistance 
together with increased triglyceride, LDL-cholesterol and RLP-cholesterol, increased VLDL and midband fractions, and increased blood glucose or glycated hemoglobin. Some reports suggest that the development of capecitabine-induced hypertriglyceridemia occurs more frequently in those with past and/or existing conditions associated with dyslipidemia, for example, obesity, hypertension, or diabetes mellitus. According to the U.S. Food \& Drug Administration, the incidence of treatment-related grade 3-4 hypertriglyceridemia is $0.1-0.2 \%$ for capecitabine as monotherapy or as part of combination therapy. Although capecitabine-induced metabolic abnormality is a rare complication, it is possible that one of the mechanisms could be a combination of various metabolic abnormalities as observed in this patient.

There is a limitation in this study. Since we started rosuvastatin before starting course 6 of the chemotherapy, we think that the decrease of LDL-cholesterol was affected by statin therapy. We thought that it would be better not to start statin in order to clearly show the influence of capecitabine on lipid and glucose metabolism but that it would be quite problematic from the ethical point of view not to start any treatment for severe dyslipidemia. Moreover, capecitabine belongs to fluorinated pyrimidine derivatives, and it is possible that fluorinated pyrimidine derivatives increased oxidative stress, which led to metabolic abnormalities in this subject. If we had measured oxidative stress markers such as oxidized LDL, it would have helped us to strengthen such hypotheses.

Taken together, we should bear in mind that capecitabine therapy could bring out not only hypertriglyceridemia but also a wide range of abnormal lipid and glucose metabolism such as hyperinsulinemia, reduction of LPL activity and increase of

\section{REFERENCES}

1. Kurt M, Babaoglu MO, Yasar U, Shorbagi A, Guler N. Capecitabine-Induced Severe Hypertriglyceridemia: Report of Two Cases. Ann Pharmacother (2006) 40:328-31. doi: 10.1345/aph.1G348

2. Koutras AK, Habeos IG, Vagenakis AG, Kalofonos HP. Capecitabine-Induced Hypertriglyceridemia: A Report of Two Cases. Anticancer Res (2006) 26:2249-51.

3. Bar-Sela G, Haim N. Uncontrolled Hypertriglyceridemia Induced by Capecitabine: Case Report and Review of the Literature. Cancer Chemother Pharmacol (2009) 63:779-82. doi: 10.1007/s00280-008-0799-2

4. Orphanos GS, Stavrou NG, Picolos MK. Hypertriglyceridemia: An Underdiagnosed Side Effect of Capecitabine Chemotherapy. Acta Oncol (2010) 49:262-3. doi: 10.3109/02841860903373708

5. Seminara P, Losanno T, Emiliani A, Manna G. Cancer Chemotherapy and Cardiovascular Risks: Is Capecitabine-Induced Hypertriglyceridemia a Rare Adverse Effect? Cardiology (2010) 116:42-4. doi: 10.1159/000313517

6. Javot L, Spaëth D, Scala-Bertola J, Gambier N, Petitpain N, Gillet P. Severe Hypertriglyceridaemia During Treatment With Capecitabine. $\mathrm{Br} J$ Cancer (2011) 104:1238-9. doi: 10.1038/bjc.2011.52

7. Polinder-Bos HA, Kok EE, van de Wiel A, Spiering W, Wielders JP, Bloemendal HJ. Severe Hypertriglyceridaemia Associated With the Use of Capecitabine. Neth J Med (2012) 70:104.

8. Leung J, Brady JL, Crook MA. The Clinical Importance of Recognizing CapecitabineInduced Hypertriglyceridemia: A Case Report and Review of the Literature. J Clin Lipidol (2018) 12:1371-3. doi: 10.1016/j.jacl.2018.07.005

9. Uche A, Vankina R, Gong J, Cho M, Yeh JJ, Kim P, et al. CapecitabineInduced Hypertriglyceridemia: A Rare But Clinically Relevant Treatment-
LDL-cholesterol. In addition, we think that this case is very important because already reported capecitabine-induced hypertriglyceridemia could be one of a wide range of abnormalities in lipid and glucose metabolism accompanied by hyperinsulinemia, insulin resistance, decreased LPL activity and hypercholesterolemia. Therefore, we have to check various lipid and glucose metabolic disorders during the period of treatment with capecitabine.

\section{DATA AVAILABILITY STATEMENT}

The raw data supporting the conclusions of this article will be made available by the authors, without undue reservation.

\section{ETHICS STATEMENT}

Written informed consent was obtained from the individual(s) for the publication of any potentially identifiable images or data included in this article.

\section{AUTHOR CONTRIBUTIONS}

TA researched data and wrote the manuscript. TY, KT, and SN researched data and contributed to the discussion. HK reviewed the manuscript. All authors contributed to the article and approved the submitted version.
Related Adverse Event. J Gastrointest Oncol (2018) 9:1213-9. doi: 10.21037/ jgo.2018.07.07

10. Chan HY, Ng CM, Tiu SC, Chan AO, Shek CC. HypertriglyceridaemiaInduced Pancreatitis: A Contributory Role of Capecitabine? Hong Kong Med J (2012) 18:526-9.

11. Scherer J, Singh VP, Pitchumoni CS, Yadav D. Issues in Hypertriglyceridemic Pancreatitis: An Update. J Clin Gastroenterol (2014) 48:195-203. doi: 10.1097/ 01.mcg.0000436438.60145.5a

12. Cornejo-Uixeda S, Escoin-Pérez C, Hernandez-Lorente E. CapecitabineInduced Pancreatitits. Med Clin (Barc) (2018) 150:e37-8. doi: 10.1016/ j.medcli.2017.10.004

13. Garg R, Angus E, Fincher S. Capecitabine-Induced Severe Hypertriglyceridaemia and Diabetes: A Case Report and Review of the Literature. Diabetes Med (2009) 26:1308-9. doi: 10.1111/j.1464-5491.2009.02842.x

14. Duman BB, Paydas S, Tetiker T, Gunaldı M, Afsar CU, Erçolak V, et al. Capecitabine-Induced Hypertriglyceridemia and Hyperglycemia: Two Cases. Pharmacology (2012) 90:212-5. doi: 10.1159/000342382

15. Han GH, Huang JX. Hypertriglyceridemia and Hyperglycemia Induced by Capecitabine: A Report of Two Cases and Review of the Literature. J Oncol Pharm Pract (2015) 21:380-3. doi: 10.1177/1078155214532508

16. Saiki A, Oyama T, Endo K, Ebisuno M, Ohira M, Koide N, et al. Preheparin Serum Lipoprotein Lipase Mass Might be a Biomarker of Metabolic Syndrome. Diabetes Res Clin Pract (2007) 76:93-101. doi: 10.1016/j.diabres.2006.08.004

17. Masuda D, Yamashita S. Postprandial Hyperlipidemia and Remnant Lipoproteins. J Atheroscler Thromb (2017) 24:95-109. doi: 10.5551/ jat.RV16003

18. Sato I, Taniguchi T, Ishikawa Y, Kusuki M, Hayashi F, Mukai M, et al. The Lipoprotein Fraction Between VLDL and LDL Detected by Biphasic 
Agarose Gel Electrophoresis Reflects Serum Remnant Lipoprotein and Lp(a) Concentrations. J Atheroscler Thromb (2006) 13:55-61. doi: 10.5551/jat.13.55

Conflict of Interest: The authors declare that the research was conducted in the absence of any commercial or financial relationships that could be construed as a potential conflict of interest.
Copyright () 2021 Anno, Yamatsuji, Tomoda, Nakanishi and Kaneto. This is an openaccess article distributed under the terms of the Creative Commons Attribution License (CC BY). The use, distribution or reproduction in other forums is permitted, provided the original author(s) and the copyright owner(s) are credited and that the original publication in this journal is cited, in accordance with accepted academic practice. No use, distribution or reproduction is permitted which does not comply with these terms. 